\title{
The Jacobi identity for graded-commutative variational Schouten bracket revisited
}

\author{
A. V. Kiselev \\ E-mail: A.V.Kiselev@rug.nl
}

\begin{abstract}
This short note contains an explicit proof of the Jacobi identity for variational Schouten bracket in $\mathbb{Z}_{2}$-graded commutative setup; an extension of the reasoning and assertion to the noncommutative geometry of cyclic words (see [1]) is immediate, still making the proof longer. We emphasize that for the reasoning to be rigorous, it must refer to the product bundle geometry of iterated variations (see [2]); on the other hand, no ad hoc regularizations occur anywhere in this theory.
\end{abstract}

Introduction. The Jacobi identity for variational Schouten bracket $\llbracket$, 』 is its key property in several cohomological theories. For example, one infers that the BV-Laplacian $\Delta$ or quantum BV-operator $\boldsymbol{\Omega}^{\hbar}=\boldsymbol{i} \hbar \Delta+\llbracket \boldsymbol{S}^{\hbar}, \cdot \rrbracket$ are differentials in the Batalin-Vilkovisky formalism (available literature is immense; let us refer to [2] and [3]) or one deduces that $\partial_{\mathcal{P}}=\llbracket \mathcal{P}, \cdot \rrbracket$ yields the Poisson-Lichnerowicz complex for every variational Poisson bi-vector $\mathcal{P}$, see [1]. Likewise, a realization of zero-curvature geometry for the inverse scattering via the classical master-equation $\llbracket \boldsymbol{S}, \boldsymbol{S} \rrbracket=0$ opens a way for deformation quantization, which is not restricted to the BV-quantization of Chern-Simons models over threefolds 1 Therefore, it is mandatory to have a clear vision of the geometry of iterated variations and understand the mechanism for validity of the Jacobi identity.

A self-regularized calculus of variations, including the definitions of $\Delta$ and $\llbracket$, and a rigorous proof of their interrelations, is developed in [2]. We reserved that theory's key element, the proof of Theorem 4.(iii) with Jacobi's identity for $\llbracket$, 』, to a separate paper which is this note. Referring to [2] for detail and discussion, let us recall that -in a theory of variations for fields over the space-time - each integral functional2 or every test shift of the fields brings its own copy of the domain of integration into the setup; the locality of couplings between (co)vectors attached at the domains' points ensures a restriction to diagonals in the accumulated products of bundles, whereas the operational definitions of $\Delta$ and $\llbracket, \rrbracket$ are on-the-diagonal reconfigurations of such couplings 3 We expect that the

\footnotetext{
${ }^{1}$ In fact, all these BV-, Poisson, or IST models are examples of variational Lie algebroids [4] and their encoding by $\boldsymbol{Q}^{2}=0$. The construction of gauge automorphisms for the $\boldsymbol{Q}$-cohomology determines the next generation of such structures, with new deformation quantization parameters beyond the Planck constant.

${ }^{2}$ Let all functionals that take field configurations to number be integral in this note; formal (sums of) products of functionals such as $\exp \left(\frac{i}{\hbar} S^{\hbar}\right)$ are dealt with by using the Leibniz rule, see [2, $\left.\S 2.5\right]$.

${ }^{3}$ It is readily seen from the proof of theorem below and from example on $\mathrm{p}$. [i that composite-structure objects such as brackets of functionals retain a kind of memory of the way how they were produced; in effect, variational derivatives detect the traces of original objects' individual geometries, whence a variation within one of them does not mar any of the others.
} 
reader is familiar with the concept and notation from $\S 1-2.4$ in [2]. In particular, we let the notation for total derivatives which stem from integrations by parts keep track of the variations' arguments, so that $((\delta s) \overleftarrow{\partial} / \partial \boldsymbol{y})(\boldsymbol{y}) \cdot \vec{\partial} \mathcal{L}\left(\boldsymbol{x},[\boldsymbol{q}],\left[\boldsymbol{q}^{\dagger}\right]\right) / \partial \boldsymbol{q}_{\boldsymbol{x}}$ at $\boldsymbol{y}=\boldsymbol{x}$ becomes $\delta s(\boldsymbol{y}) \cdot(-\overrightarrow{\mathrm{d}} / \mathrm{d} \boldsymbol{y})\left(\vec{\partial} \mathcal{L}\left(\boldsymbol{x},[\boldsymbol{q}],\left[\boldsymbol{q}^{\dagger}\right]\right) / \partial \boldsymbol{q}_{\boldsymbol{x}}\right)$ on that diagonal, see Example on p. il and Example 2.4 on pp. 34-36 of [2]. Similarly, the variational derivatives with respect to (anti)fields $\boldsymbol{q}$ or $\boldsymbol{q}^{\dagger}$ keep track of the test shifts which those variations come from: e.g., the formula above yields 4 a term in $\delta s(\boldsymbol{y}) \cdot \vec{\delta} / \delta \boldsymbol{q}(\boldsymbol{y})\left(\mathcal{L}\left(\boldsymbol{x},[\boldsymbol{q}],\left[\boldsymbol{q}^{\dagger}\right]\right)\right)$ at $\boldsymbol{y}=\boldsymbol{x}$. This simplifies the reasoning 5

Theorem. Let $F, G$, and $H$ be $\mathbb{Z}_{2}$-parity homogeneous functionals; denote by $|\cdot|$ the grading so that $(-)^{|\cdot|}$ is the parity. The variational Schouten bracket $\llbracket$, satisfies the shifted-graded Jacobi identity (cf. Eq. (28) in Theorem 4.(iii) on p. 30 versus Eq. (36) on p. 37 in [2]),

$$
\llbracket F, \llbracket G, H \rrbracket \rrbracket=\llbracket \llbracket F, G \rrbracket, H \rrbracket+(-)^{(|F|-1)(|G|-1)} \llbracket G, \llbracket F, H \rrbracket \rrbracket .
$$

The operator $\llbracket F, \cdot \rrbracket$ is a graded derivation of $\llbracket, \rrbracket$ : identity (11) is the Leibniz rule for it.

Proof. The logic is straightforward $\sqrt{6}$ as soon as the matching of (co)vectors and reconfigurations of couplings are understood in [2, $\S 1-2]$. We consider first the 1.-h.s. of (11). By construction, we have that $\llbracket G, H \rrbracket=\left(G\left(\boldsymbol{x}_{2}\right)\right) \overleftarrow{\delta} / \delta \boldsymbol{q}\left(\boldsymbol{y}_{2}\right) \cdot \vec{\delta} / \delta \boldsymbol{q}^{\dagger}\left(\boldsymbol{y}_{3}\right)\left(H\left(\boldsymbol{x}_{3}\right)\right)-\left(G\left(\boldsymbol{x}_{2}\right)\right) \overleftarrow{\delta} / \delta \boldsymbol{q}^{\dagger}\left(\boldsymbol{y}_{2}\right)$ $\vec{\delta} / \delta \boldsymbol{q}\left(\boldsymbol{y}_{3}\right)\left(H\left(\boldsymbol{x}_{3}\right)\right)$. Now expanding $\llbracket F, \llbracket G, H \rrbracket \rrbracket=\left(F\left(\boldsymbol{x}_{1}\right)\right) \overleftarrow{\delta} / \delta \boldsymbol{q}\left(\boldsymbol{z}_{1}\right) \cdot \vec{\delta} / \delta \boldsymbol{q}^{\dagger}\left(\boldsymbol{z}_{23}\right)(\llbracket G, H \rrbracket)-$ $\left(F\left(\boldsymbol{x}_{1}\right)\right) \overleftarrow{\delta} / \delta \boldsymbol{q}^{\dagger}\left(\boldsymbol{z}_{1}\right) \cdot \vec{\delta} / \delta \boldsymbol{q}\left(\boldsymbol{z}_{23}\right)(\llbracket G, H \rrbracket)$, we obtain the sum of eight enumerated terms 7

$$
\begin{aligned}
& \langle 1\rangle F\left(\boldsymbol{x}_{1}\right) \overleftarrow{\delta} / \delta \boldsymbol{q}\left(\boldsymbol{z}_{1}\right) \cdot \vec{\delta} / \delta \boldsymbol{q}^{\dagger}\left(\boldsymbol{z}_{\mathbf{2} 3}\right) G\left(\boldsymbol{x}_{2}\right) \overleftarrow{\delta} / \delta \boldsymbol{q}\left(\boldsymbol{y}_{2}\right) \cdot \vec{\delta} / \delta \boldsymbol{q}^{\dagger}\left(\boldsymbol{y}_{3}\right) H\left(\boldsymbol{x}_{3}\right)+ \\
& \langle 2\rangle+(-)^{|G|} F\left(\boldsymbol{x}_{1}\right) \overleftarrow{\delta} / \delta \boldsymbol{q}\left(\boldsymbol{z}_{1}\right) \cdot G\left(\boldsymbol{x}_{2}\right) \overleftarrow{\delta} \delta \boldsymbol{q}\left(\boldsymbol{y}_{2}\right) \cdot \vec{\delta} / \delta \boldsymbol{q}^{\dagger}\left(\boldsymbol{z}_{23}\right) \vec{\delta} / \delta \boldsymbol{q}^{\dagger}\left(\boldsymbol{y}_{3}\right) H\left(\boldsymbol{x}_{3}\right)- \\
& \langle 3\rangle \quad-F\left(\boldsymbol{x}_{1}\right) \overleftarrow{\delta} / \delta \boldsymbol{q}\left(\boldsymbol{z}_{1}\right) \cdot \vec{\delta} / \delta \boldsymbol{q}^{\dagger}\left(\boldsymbol{z}_{\mathbf{2} 3}\right)\left(G\left(\boldsymbol{x}_{2}\right) \overleftarrow{\delta} / \delta \boldsymbol{q}^{\dagger}\left(\boldsymbol{y}_{2}\right)\right) \cdot \vec{\delta} / \delta \boldsymbol{q}\left(\boldsymbol{y}_{3}\right) H\left(\boldsymbol{x}_{3}\right)- \\
& \langle 4\rangle \quad-(-)^{|G|-1} F\left(\boldsymbol{x}_{1}\right) \overleftarrow{\delta} / \delta \boldsymbol{q}\left(\boldsymbol{z}_{1}\right) \cdot G\left(\boldsymbol{x}_{2}\right) \overleftarrow{\delta} / \delta \boldsymbol{q}^{\dagger}\left(\boldsymbol{y}_{2}\right) \cdot \vec{\delta} / \delta \boldsymbol{q}^{\dagger}\left(\boldsymbol{z}_{23}\right) \vec{\delta} / \delta \boldsymbol{q}\left(\boldsymbol{y}_{3}\right) H\left(\boldsymbol{x}_{3}\right)- \\
& \langle 5\rangle \quad-F\left(\boldsymbol{x}_{1}\right) \overleftarrow{\delta} / \delta \boldsymbol{q}^{\dagger}\left(\boldsymbol{z}_{1}\right) \cdot \vec{\delta} / \delta \boldsymbol{q}\left(\boldsymbol{z}_{\mathbf{2} 3}\right) G\left(\boldsymbol{x}_{2}\right) \overleftarrow{\delta} / \delta \boldsymbol{q}\left(\boldsymbol{y}_{2}\right) \cdot \vec{\delta} / \delta \boldsymbol{q}^{\dagger}\left(\boldsymbol{y}_{3}\right) H\left(\boldsymbol{x}_{3}\right)- \\
& \langle 6\rangle \quad-F\left(\boldsymbol{x}_{1}\right) \overleftarrow{\delta} / \delta \boldsymbol{q}^{\dagger}\left(\boldsymbol{z}_{1}\right) \cdot G\left(\boldsymbol{x}_{2}\right) \overleftarrow{\delta} / \delta \boldsymbol{q}\left(\boldsymbol{y}_{2}\right) \cdot \vec{\delta} / \delta \boldsymbol{q}\left(\boldsymbol{z}_{23}\right) \vec{\delta} / \delta \boldsymbol{q}^{\dagger}\left(\boldsymbol{y}_{3}\right) H\left(\boldsymbol{x}_{3}\right)+ \\
& \langle 7\rangle \quad+F\left(\boldsymbol{x}_{1}\right) \overleftarrow{\delta} / \delta \boldsymbol{q}^{\dagger}\left(\boldsymbol{z}_{1}\right) \cdot \vec{\delta} / \delta \boldsymbol{q}\left(\boldsymbol{z}_{\mathbf{2} 3}\right) G\left(\boldsymbol{x}_{2}\right) \overleftarrow{\delta} / \delta \boldsymbol{q}^{\dagger}\left(\boldsymbol{y}_{2}\right) \cdot \vec{\delta} / \delta \boldsymbol{q}\left(\boldsymbol{y}_{3}\right) H\left(\boldsymbol{x}_{3}\right)+ \\
& \langle 8\rangle+F\left(\boldsymbol{x}_{1}\right) \overleftarrow{\delta} / \delta \boldsymbol{q}^{\dagger}\left(\boldsymbol{z}_{1}\right) \cdot G\left(\boldsymbol{x}_{2}\right) \overleftarrow{\delta} / \delta \boldsymbol{q}^{\dagger}\left(\boldsymbol{y}_{2}\right) \cdot \vec{\delta} / \delta \boldsymbol{q}\left(\boldsymbol{z}_{23}\right) \vec{\delta} / \delta \boldsymbol{q}\left(\boldsymbol{y}_{3}\right) H\left(\boldsymbol{x}_{3}\right)
\end{aligned}
$$

Arguing as above, we see that the term $\llbracket \llbracket F, G \rrbracket, H \rrbracket$ in the r.-h.s. of (11) is 8

\footnotetext{
${ }^{4}$ In this note we let the arrow over a variational derivative indicate the direction along which all derivatives act - but not the opposite direction along which the test shifts were transported prior to any integration by parts (cf. 2]); we thus have $\overrightarrow{\delta \boldsymbol{s}}(\boldsymbol{S})=\int \mathrm{d} \boldsymbol{y}\left\{\langle\delta s(\boldsymbol{y}), \vec{\delta} / \delta \boldsymbol{q}(\boldsymbol{y})(\boldsymbol{S}(\boldsymbol{x}))\rangle+\left\langle\delta s^{\dagger}(\boldsymbol{y}), \vec{\delta} / \delta \boldsymbol{q}^{\dagger}(\boldsymbol{y})(\boldsymbol{S}(\boldsymbol{x}))\right\rangle\right\}$ and $(\boldsymbol{S}) \overleftarrow{\delta \boldsymbol{s}}=\int \mathrm{d} \boldsymbol{y}\left\{\langle(\boldsymbol{S}(\boldsymbol{x})) \overleftarrow{\delta} / \delta \boldsymbol{q}(\boldsymbol{y}), \delta s(\boldsymbol{y})\rangle+\left\langle(\boldsymbol{S}(\boldsymbol{x})) \overleftarrow{\delta} / \delta \boldsymbol{q}^{\dagger}(\boldsymbol{y}), \delta s^{\dagger}(\boldsymbol{y})\right\rangle\right\}$, where the diagonal $\boldsymbol{y}=\boldsymbol{x}$ is wrought by the coupling $\langle$,$\rangle , see [2, \S 2.2-3]$, and we display the integration variable $\boldsymbol{x}$ in the functional $\boldsymbol{S}$.

${ }^{5}$ With a bit more care taken of the order in which the factors follow each other in products, and by using the $\mathbb{Z}_{2}$-graded Leibniz rule for left- and right-directed derivations, we show that the claim and proof of the main theorem hold true in the setup of cyclic words and brackets of necklaces (see [1] and references therein).

${ }^{6}$ Obviously, the l.-h.s. of (1) does not contain second variational derivatives of $F$ whereas the r.-h.s. does. We show that it is precisely these terms and none others which cancel out in the r.-h.s.

${ }^{7}$ We denote by $\boldsymbol{z}_{i j}$ the integration variables which label the variations falling - in the outer brackets in (1) - on the $i$ th or $j$ th functional by the Leibniz rule (let $F$ be first and so on, $1 \leqslant i<j \leqslant 3$ ); for convenience, we highlight $i$ in $\boldsymbol{z}_{i j}$ when the variation falls on the $i$ th functional - and $j$ otherwise.

${ }^{8}$ The labelling of terms by superscripts $\langle 1\rangle-\langle 8\rangle$ shows their matching with summands in the l.-h.s. of (1) or, for the index running from $\langle 9\rangle$ to $\langle 12\rangle$, points at the four second-order variations of $F$ which cancel out in the two r.-h.s. summands in Jacobi's identity.
} 


$$
\begin{aligned}
& { }^{\langle 9\rangle} F\left(\boldsymbol{x}_{1}\right) \overleftarrow{\delta} / \delta \boldsymbol{q}\left(\boldsymbol{y}_{1}\right) \overleftarrow{\delta} / \delta \boldsymbol{q}\left(\boldsymbol{z}_{12}\right) \cdot \vec{\delta} / \delta \boldsymbol{q}^{\dagger}\left(\boldsymbol{y}_{2}\right) G\left(\boldsymbol{x}_{2}\right) \cdot \vec{\delta} / \delta \boldsymbol{q}^{\dagger}\left(\boldsymbol{z}_{3}\right) H\left(\boldsymbol{x}_{3}\right)+ \\
& \langle 1\rangle \quad+F\left(\boldsymbol{x}_{1}\right) \overleftarrow{\delta} / \delta \boldsymbol{q}\left(\boldsymbol{y}_{1}\right) \cdot \vec{\delta} / \delta \boldsymbol{q}^{\dagger}\left(\boldsymbol{y}_{2}\right) G\left(\boldsymbol{x}_{2}\right) \overleftarrow{\delta} / \delta \boldsymbol{q}\left(\boldsymbol{z}_{12}\right) \cdot \vec{\delta} / \delta \boldsymbol{q}^{\dagger}\left(\boldsymbol{z}_{3}\right) H\left(\boldsymbol{x}_{3}\right)- \\
& \langle 10\rangle \quad-F\left(\boldsymbol{x}_{1}\right) \overleftarrow{\delta} / \delta \boldsymbol{q}^{\dagger}\left(\boldsymbol{y}_{1}\right) \overleftarrow{\delta} / \delta \boldsymbol{q}\left(\boldsymbol{z}_{12}\right) \cdot \vec{\delta} / \delta \boldsymbol{q}\left(\boldsymbol{y}_{2}\right) G\left(\boldsymbol{x}_{2}\right) \cdot \vec{\delta} / \delta \boldsymbol{q}^{\dagger}\left(\boldsymbol{z}_{3}\right) H\left(\boldsymbol{x}_{3}\right)- \\
& \langle 5\rangle \quad-F\left(\boldsymbol{x}_{1}\right) \overleftarrow{\delta} / \delta \boldsymbol{q}^{\dagger}\left(\boldsymbol{y}_{1}\right) \cdot \vec{\delta} / \delta \boldsymbol{q}\left(\boldsymbol{y}_{2}\right) G\left(\boldsymbol{x}_{2}\right) \overleftarrow{\delta / \delta} \boldsymbol{q}\left(\boldsymbol{z}_{12}\right) \cdot \vec{\delta} / \delta \boldsymbol{q}^{\dagger}\left(\boldsymbol{z}_{3}\right) H\left(\boldsymbol{x}_{3}\right)- \\
& \langle 11\rangle \quad-(-)^{|G|-1} F\left(\boldsymbol{x}_{1}\right) \overleftarrow{\delta} / \delta \boldsymbol{q}\left(\boldsymbol{y}_{1}\right) \overleftarrow{\delta} / \delta \boldsymbol{q}^{\dagger}\left(\boldsymbol{z}_{12}\right) \cdot \vec{\delta} / \delta \boldsymbol{q}^{\dagger}\left(\boldsymbol{y}_{2}\right) G\left(\boldsymbol{x}_{2}\right) \cdot \vec{\delta} / \delta \boldsymbol{q}\left(\boldsymbol{z}_{3}\right) H\left(\boldsymbol{x}_{3}\right)- \\
& \langle 3\rangle-F\left(\boldsymbol{x}_{1}\right) \overleftarrow{\delta} / \delta \boldsymbol{q}\left(\boldsymbol{y}_{1}\right) \cdot\left(\vec{\delta} / \delta \boldsymbol{q}^{\dagger}\left(\boldsymbol{y}_{2}\right) G\left(\boldsymbol{x}_{2}\right)\right) \overleftarrow{\delta} / \delta \boldsymbol{q}^{\dagger}\left(\boldsymbol{z}_{12}\right) \cdot \vec{\delta} / \delta \boldsymbol{q}\left(\boldsymbol{z}_{3}\right) H\left(\boldsymbol{x}_{3}\right)+ \\
& \langle 12\rangle+(-)^{|G|} F\left(\boldsymbol{x}_{1}\right) \overleftarrow{\delta} \delta \boldsymbol{q}^{\dagger}\left(\boldsymbol{y}_{1}\right) \overleftarrow{\delta} / \delta \boldsymbol{q}^{\dagger}\left(\boldsymbol{z}_{12}\right) \cdot \vec{\delta} / \delta \boldsymbol{q}\left(\boldsymbol{y}_{2}\right) G\left(\boldsymbol{x}_{2}\right) \cdot \vec{\delta} / \delta \boldsymbol{q}\left(\boldsymbol{z}_{3}\right) H\left(\boldsymbol{x}_{3}\right)+ \\
& \langle 7\rangle \quad+F\left(\boldsymbol{x}_{1}\right) \overleftarrow{\delta} / \delta \boldsymbol{q}^{\dagger}\left(\boldsymbol{y}_{1}\right) \cdot \vec{\delta} / \delta \boldsymbol{q}\left(\boldsymbol{y}_{2}\right) G\left(\boldsymbol{x}_{2}\right) \overleftarrow{\delta} / \delta \boldsymbol{q}^{\dagger}\left(\boldsymbol{z}_{12}\right) \cdot \vec{\delta} / \delta \boldsymbol{q}\left(\boldsymbol{z}_{3}\right) H\left(\boldsymbol{x}_{3}\right)
\end{aligned}
$$

In the same way, we obtain the term $\llbracket G, \llbracket F, H \rrbracket \rrbracket$ not yet multiplied by the extra sign factor:

$$
\begin{aligned}
& { }^{\{1\}} G\left(\boldsymbol{x}_{2}\right) \overleftarrow{\delta} / \delta \boldsymbol{q}\left(\boldsymbol{z}_{2}\right) \cdot \vec{\delta} / \delta \boldsymbol{q}^{\dagger}\left(\boldsymbol{z}_{13}\right) F\left(\boldsymbol{x}_{1}\right) \overleftarrow{\delta} / \delta \boldsymbol{q}\left(\boldsymbol{y}_{1}\right) \cdot \vec{\delta} / \delta \boldsymbol{q}^{\dagger}\left(\boldsymbol{y}_{3}\right) H\left(\boldsymbol{x}_{3}\right)+ \\
& { }^{22\}}+(-)^{|F|} G\left(\boldsymbol{x}_{2}\right) \overleftarrow{\delta} / \delta \boldsymbol{q}\left(\boldsymbol{z}_{2}\right) \cdot F\left(\boldsymbol{x}_{1}\right) \overleftarrow{\delta} / \delta \boldsymbol{q}\left(\boldsymbol{y}_{1}\right) \cdot \vec{\delta} / \delta \boldsymbol{q}^{\dagger}\left(\boldsymbol{z}_{1 \mathbf{3}}\right) \vec{\delta} / \delta \boldsymbol{q}^{\dagger}\left(\boldsymbol{y}_{3}\right) H\left(\boldsymbol{x}_{3}\right)- \\
& { }^{\{3\}}-G\left(\boldsymbol{x}_{2}\right) \overleftarrow{\delta} / \delta \boldsymbol{q}\left(\boldsymbol{z}_{2}\right) \cdot \vec{\delta} / \delta \boldsymbol{q}^{\dagger}\left(\boldsymbol{z}_{13}\right)\left(F\left(\boldsymbol{x}_{1}\right) \overleftarrow{\delta} / \delta \boldsymbol{q}^{\dagger}\left(\boldsymbol{y}_{1}\right)\right) \cdot \vec{\delta} / \delta \boldsymbol{q}\left(\boldsymbol{y}_{3}\right) H\left(\boldsymbol{x}_{3}\right)- \\
& \{4\} \quad-(-)^{|F|-1} G\left(\boldsymbol{x}_{2}\right) \overleftarrow{\delta} / \delta \boldsymbol{q}\left(\boldsymbol{z}_{2}\right) \cdot F\left(\boldsymbol{x}_{1}\right) \overleftarrow{\delta} / \delta \boldsymbol{q}^{\dagger}\left(\boldsymbol{y}_{1}\right) \cdot \vec{\delta} / \delta \boldsymbol{q}^{\dagger}\left(\boldsymbol{z}_{13}\right) \vec{\delta} / \delta \boldsymbol{q}\left(\boldsymbol{y}_{3}\right) H\left(\boldsymbol{x}_{3}\right)- \\
& \{5\}-G\left(\boldsymbol{x}_{2}\right) \overleftarrow{\delta} / \delta \boldsymbol{q}^{\dagger}\left(\boldsymbol{z}_{2}\right) \cdot \vec{\delta} / \delta \boldsymbol{q}\left(\boldsymbol{z}_{13}\right) F\left(\boldsymbol{x}_{1}\right) \overleftarrow{\delta} / \delta \boldsymbol{q}\left(\boldsymbol{y}_{1}\right) \cdot \vec{\delta} / \delta \boldsymbol{q}^{\dagger}\left(\boldsymbol{y}_{3}\right) H\left(\boldsymbol{x}_{3}\right)- \\
& \{6\} \quad-G\left(\boldsymbol{x}_{2}\right) \overleftarrow{\delta} / \delta \boldsymbol{q}^{\dagger}\left(\boldsymbol{z}_{2}\right) \cdot F\left(\boldsymbol{x}_{1}\right) \overleftarrow{\delta} / \delta \boldsymbol{q}\left(\boldsymbol{y}_{1}\right) \cdot \vec{\delta} / \delta \boldsymbol{q}\left(\boldsymbol{z}_{13}\right) \vec{\delta} / \delta \boldsymbol{q}^{\dagger}\left(\boldsymbol{y}_{3}\right) H\left(\boldsymbol{x}_{3}\right)+ \\
& \{7\} \quad+G\left(\boldsymbol{x}_{2}\right) \overleftarrow{\delta} / \delta \boldsymbol{q}^{\dagger}\left(\boldsymbol{z}_{2}\right) \cdot \vec{\delta} / \delta \boldsymbol{q}\left(\boldsymbol{z}_{13}\right) F\left(\boldsymbol{x}_{1}\right) \overleftarrow{\delta} / \delta \boldsymbol{q}^{\dagger}\left(\boldsymbol{y}_{1}\right) \cdot \vec{\delta} / \delta \boldsymbol{q}\left(\boldsymbol{y}_{3}\right) H\left(\boldsymbol{x}_{3}\right)+ \\
& \{8\}+G\left(\boldsymbol{x}_{2}\right) \overleftarrow{\delta} / \delta \boldsymbol{q}^{\dagger}\left(\boldsymbol{z}_{2}\right) \cdot F\left(\boldsymbol{x}_{1}\right) \overleftarrow{\delta} / \delta \boldsymbol{q}^{\dagger}\left(\boldsymbol{y}_{1}\right) \cdot \vec{\delta} / \delta \boldsymbol{q}\left(\boldsymbol{z}_{13}\right) \vec{\delta} / \delta \boldsymbol{q}\left(\boldsymbol{y}_{3}\right) H\left(\boldsymbol{x}_{3}\right)
\end{aligned}
$$

Let us now use the $\mathbb{Z}_{2}$-graded commutativity assumption for the setup. Transporting the variations of $F$ leftmost, we restore the lexicographic order $F \prec G \prec H$. Finally, we multiply $\llbracket G, \llbracket F, H, \rrbracket \rrbracket$, reordered as above, by the sign factor $(-)^{(|F|-1)(|G|-1)}$; this yields $₫$

$$
\begin{aligned}
& { }^{\langle 10\rangle}(-)^{|F|-1} \vec{\delta} / \delta \boldsymbol{q}^{\dagger}\left(\boldsymbol{z}_{13}\right) F\left(\boldsymbol{x}_{1}\right) \overleftarrow{\delta} / \delta \boldsymbol{q}\left(\boldsymbol{y}_{1}\right) \cdot G\left(\boldsymbol{x}_{2}\right) \overleftarrow{\delta} / \delta \boldsymbol{q}\left(\boldsymbol{z}_{2}\right) \cdot \vec{\delta} / \delta \boldsymbol{q}^{\dagger}\left(\boldsymbol{y}_{3}\right) H\left(\boldsymbol{x}_{3}\right)+ \\
& \langle 2\rangle \quad+(-)^{|G|-1} F\left(\boldsymbol{x}_{1}\right) \overleftarrow{\delta} / \delta \boldsymbol{q}\left(\boldsymbol{y}_{1}\right) \cdot G\left(\boldsymbol{x}_{2}\right) \overleftarrow{\delta} \delta \boldsymbol{q}\left(\boldsymbol{z}_{2}\right) \cdot \vec{\delta} / \delta \boldsymbol{q}^{\dagger}\left(\boldsymbol{z}_{13}\right) \vec{\delta} / \delta \boldsymbol{q}^{\dagger}\left(\boldsymbol{y}_{3}\right) H\left(\boldsymbol{x}_{3}\right)+ \\
& \langle 12\rangle+(-)^{|F|+|G|} \vec{\delta} / \delta \boldsymbol{q}^{\dagger}\left(\boldsymbol{z}_{13}\right)\left(F\left(\boldsymbol{x}_{1}\right) \overleftarrow{\delta} / \delta \boldsymbol{q}^{\dagger}\left(\boldsymbol{y}_{1}\right)\right) \cdot G\left(\boldsymbol{x}_{2}\right) \overleftarrow{\delta} / \delta \boldsymbol{q}\left(\boldsymbol{z}_{2}\right) \cdot \vec{\delta} / \delta \boldsymbol{q}\left(\boldsymbol{y}_{3}\right) H\left(\boldsymbol{x}_{3}\right)- \\
& \langle 6\rangle-F\left(\boldsymbol{x}_{1}\right) \overleftarrow{\delta} / \delta \boldsymbol{q}^{\dagger}\left(\boldsymbol{y}_{1}\right) \cdot G\left(\boldsymbol{x}_{2}\right) \overleftarrow{\delta} / \delta \boldsymbol{q}\left(\boldsymbol{z}_{2}\right) \cdot \vec{\delta} / \delta \boldsymbol{q}^{\dagger}\left(\boldsymbol{z}_{13}\right) \vec{\delta} / \delta \boldsymbol{q}\left(\boldsymbol{y}_{3}\right) H\left(\boldsymbol{x}_{3}\right)+ \\
& \langle 9\rangle \quad+(-)^{|G|} \vec{\delta} / \delta \boldsymbol{q}\left(\boldsymbol{z}_{13}\right) F\left(\boldsymbol{x}_{1}\right) \overleftarrow{\delta} / \delta \boldsymbol{q}\left(\boldsymbol{y}_{1}\right) \cdot G\left(\boldsymbol{x}_{2}\right) \overleftarrow{\delta} / \delta \boldsymbol{q}^{\dagger}\left(\boldsymbol{z}_{2}\right) \cdot \vec{\delta} / \delta \boldsymbol{q}^{\dagger}\left(\boldsymbol{y}_{3}\right) H\left(\boldsymbol{x}_{3}\right)+ \\
& { }^{\langle 4\rangle} \quad+(-)^{|G|} F\left(\boldsymbol{x}_{1}\right) \overleftarrow{\delta} / \delta \boldsymbol{q}\left(\boldsymbol{y}_{1}\right) \cdot G\left(\boldsymbol{x}_{2}\right) \overleftarrow{\delta} / \delta \boldsymbol{q}^{\dagger}\left(\boldsymbol{z}_{2}\right) \cdot \vec{\delta} / \delta \boldsymbol{q}\left(\boldsymbol{z}_{13}\right) \vec{\delta} / \delta \boldsymbol{q}^{\dagger}\left(\boldsymbol{y}_{3}\right) H\left(\boldsymbol{x}_{3}\right)+ \\
& \langle 11\rangle+\vec{\delta} / \delta \boldsymbol{q}\left(\boldsymbol{z}_{13}\right) F\left(\boldsymbol{x}_{1}\right) \overleftarrow{\delta} / \delta \boldsymbol{q}^{\dagger}\left(\boldsymbol{y}_{1}\right) \cdot G\left(\boldsymbol{x}_{2}\right) \overleftarrow{\delta} / \delta \boldsymbol{q}^{\dagger}\left(\boldsymbol{z}_{2}\right) \cdot \vec{\delta} / \delta \boldsymbol{q}\left(\boldsymbol{y}_{3}\right) H\left(\boldsymbol{x}_{3}\right)+ \\
& \langle 8\rangle+F\left(\boldsymbol{x}_{1}\right) \overleftarrow{\delta} / \delta \boldsymbol{q}^{\dagger}\left(\boldsymbol{y}_{1}\right) \cdot G\left(\boldsymbol{x}_{2}\right) \overleftarrow{\delta} / \delta \boldsymbol{q}^{\dagger}\left(\boldsymbol{z}_{2}\right) \cdot \vec{\delta} / \delta \boldsymbol{q}\left(\boldsymbol{z}_{13}\right) \vec{\delta} / \delta \boldsymbol{q}\left(\boldsymbol{y}_{3}\right) H\left(\boldsymbol{x}_{3}\right)
\end{aligned}
$$

\footnotetext{
${ }^{9}$ For each term labelled by $\{1\}-\{8\}$ in $\llbracket G, \llbracket F, H, \rrbracket$, let us calculate the product of three signs: one which was written near the respective summand, the other which comes from the reorderings to $F \prec G$, and thirdly, $(-)^{(|F|-1)(|G|-1)}$; here is the list: $\{1\}:(-)^{(|F|-1) \cdot|G|}(-)^{(|F|-1)(|G|-1)}=(-)^{|F|-1}$, $\{2\}:(-)^{|F|}(-)^{|F| \cdot|G|}(-)^{(|F|-1)(|G|-1)}=(-)^{|G|-1}, \quad\{3\}:-(-)^{(|F|-2) \cdot|G|}(-)^{(|F|-1)(|G|-1)}=(-)^{|F|+|G|}, \quad\{4\}:$ $-(-)^{|F|-1}(-)^{(|F|-1) \cdot|G|}(-)^{(|F|-1)(|G|-1)}=-1, \quad\{5\},\{6\}:-(-)^{|F| \cdot(|G|-1)}(-)^{(|F|-1)(|G|-1)}=(-)^{|G|}, \quad\{7\}$, $\{8\}:(-)^{(|F|-1) \cdot(|G|-1)}(-)^{(|F|-1)(|G|-1)}=+1$.
} 
Terms $\langle 1\rangle-\langle 8\rangle$ are present in the r.-h.s. of (1) and terms $\langle 9\rangle-\langle 12\rangle$ cancel out; it is only the indices $\langle 3\rangle$ and $\langle 12\rangle$ which require special attention. Consider $\langle 3\rangle$ in $\llbracket \llbracket F, G \rrbracket, H \rrbracket$; by relabelling the integration variables, $\boldsymbol{y} \rightleftarrows \boldsymbol{z}$ (i.e., swapping the test shifts, see p.il), we obtain

$$
-F\left(\boldsymbol{x}_{1}\right) \overleftarrow{\delta} / \delta \boldsymbol{q}\left(\boldsymbol{z}_{1}\right) \cdot\left(\vec{\delta} / \delta \boldsymbol{q}^{\dagger}\left(\boldsymbol{z}_{12}\right) G\left(\boldsymbol{x}_{2}\right)\right) \overleftarrow{\delta} / \delta \boldsymbol{q}^{\dagger}\left(\boldsymbol{y}_{2}\right) \cdot \vec{\delta} / \delta \boldsymbol{q}\left(\boldsymbol{y}_{3}\right) H\left(\boldsymbol{x}_{3}\right)
$$

The variation's argument in parentheses has grading $|G|-1$, which yields the sign factor $(-)^{(|G|-1)-1}$ when the left-acting parity-odd variation $\overleftarrow{\delta} / \delta \boldsymbol{q}^{\dagger}\left(\boldsymbol{y}_{2}\right)$ is brought to the other side of its argument, becoming $\vec{\delta} / \delta \boldsymbol{q}^{\dagger}\left(\boldsymbol{y}_{2}\right)$. Hence $(-)^{|G|-2} \vec{\delta} / \delta \boldsymbol{q}^{\dagger}\left(\boldsymbol{y}_{2}\right)\left(\vec{\delta} / \delta \boldsymbol{q}^{\dagger}\left(\boldsymbol{z}_{\mathbf{2} 3}\right)\left(G\left(\boldsymbol{x}_{2}\right)\right)\right) \stackrel{(i)}{=}$ $(-)^{|G|-1} \vec{\delta} / \delta \boldsymbol{q}^{\dagger}\left(\boldsymbol{z}_{\mathbf{2} 3}\right)\left(\vec{\delta} / \delta \boldsymbol{q}^{\dagger}\left(\boldsymbol{y}_{2}\right)\left(G\left(\boldsymbol{x}_{2}\right)\right)\right) \stackrel{(i i)}{=}(-)^{|G|-1}(-)^{|G|-1} \vec{\delta} / \delta \boldsymbol{q}^{\dagger}\left(\boldsymbol{z}_{\mathbf{2} 3}\right)\left(\left(G\left(\boldsymbol{x}_{2}\right)\right) \overleftarrow{\delta} / \delta \boldsymbol{q}^{\dagger}\left(\boldsymbol{y}_{2}\right)\right)$ where $(i)$ the parity-odd variations are swapped and $(i i)$ the inner variational derivative is transported around $G$ of grading $|G|$. The two sign factors cancel out, and the overall minus matches that near $\langle 3\rangle$ in the 1.-h.s. of (1).

We do the same with $\langle 12\rangle$. Consider such term in $(-)^{(|F|-1)(|G|-1)} \llbracket G, \llbracket F, H \rrbracket \rrbracket ;$ clearly, the factor $(-)^{|G|}$ is irrelevant because it is present also near $\langle 12\rangle$ in $\llbracket \llbracket F, G \rrbracket, H \rrbracket$. Transporting the parity-odd variation $\vec{\delta} / \delta \boldsymbol{q}^{\dagger}\left(\boldsymbol{z}_{13}\right)$ around the object of grading $|F|-1$ in parentheses, we gain the factor $(-)^{|F|-2}$, which cancels out with $(-)^{|F|}$. Next, relabel $\boldsymbol{y} \rightleftarrows \boldsymbol{z}$, which gives

$$
F\left(\boldsymbol{x}_{1}\right) \overleftarrow{\delta} / \delta \boldsymbol{q}^{\dagger}\left(\boldsymbol{z}_{13}\right) \overleftarrow{\delta} / \delta \boldsymbol{q}^{\dagger}\left(\boldsymbol{y}_{1}\right) \cdot G\left(\boldsymbol{x}_{2}\right) \overleftarrow{\delta} / \delta \boldsymbol{q}\left(\boldsymbol{y}_{2}\right) \cdot \vec{\delta} / \delta \boldsymbol{q}\left(\boldsymbol{z}_{1 \mathbf{3}}\right) H\left(\boldsymbol{x}_{3}\right)
$$

The parity-odd variations follow in the order which is reverse with respect to that in $\langle 12\rangle$ in $\llbracket \llbracket F, G \rrbracket, H \rrbracket$, hence these terms cancel out. The proof is complete.

Conclusion. Variations $\delta \boldsymbol{s}$ act via graded Leibniz rule on products of integral functionals, e.g., $F \cdot \llbracket G, H \rrbracket$; within composite objects like $\llbracket G, H \rrbracket$, they act also by derivation w.r.t. own geometries of the blocks $G, H$; variations are graded-permutable in each block. Neither $\Delta$ nor $\llbracket, \rrbracket$ depend on a choice of normalized test shift $\delta \boldsymbol{s}$. This yields (11) and $\Delta^{2}(F \cdot G \cdot H)=0$.

Acknowledgements. The author thanks the Organizing committee of International workshop SQS'13 'Supersymmetry and Quantum Symmetries' (July 29 - August 3, 2013; JINR Dubna, Russia) for stimulating discussions and partial financial support.

This research was supported in part by JBI RUG project 103511 (Groningen). A part of this research was done while the author was visiting at the IHÉS (Bures-sur-Yvette); the financial support and hospitality of this institution are gratefully acknowledged.

\section{References}

[1] Kiselev A. V. On the variational noncommutative Poisson geometry // Physics of Particles and Nuclei. 2012. V. 43, n.5. P. 663-665.

[2] Kiselev A. V. The geometry of variations in Batalin-Vilkovisky formalism // J. Phys.: Conf. Ser. 2013. V. 474, n.012024. P. 1-51.

[3] Batalin I., Vilkovisky G. Gauge algebra and quantization // Phys. Lett. B. 1981. V. 102, n.1. P. 27-31; Batalin I. A., Vilkovisky G. A. Quantization of gauge theories with linearly dependent generators // Phys. Rev. D. 1983. V. 29, n.10. P. 2567-2582.

[4] Kiselev A. V., van de Leur J. W. Variational Lie algebroids and homological evolutionary vector fields // Theor. Math. Phys. 2011. V. 167, n.3. P. 772-784. 
Appendix: an example. Let us illustrate the validity mechanism for Jacobi identity (1) by verifying it at three given functionals. For simplicity, let there be just one independent variable $x$, one parity-even coordinate $q$ and its parity-odd canonically conjugate $q^{\dagger}$. Set

$$
F=\int q^{\dagger} q q_{x_{1} x_{1}} \operatorname{dvol}\left(x_{1}\right), \quad G=\int q_{x_{2}}^{\dagger} \exp \left(q_{x_{2}}\right) \operatorname{dvol}\left(x_{2}\right), \quad \text { and } H=\int q_{x_{3} x_{3}}^{\dagger} \cos q \operatorname{dvol}\left(x_{3}\right) ;
$$

we note that the functionals $F$ and $H$ re-appear in [2, pp. 34-36]. We have $|F|=1$ and $|G|=1$, whence $(-)^{(|F|-1)(|G|-1)}=+1$ in (1).

Let $\delta \boldsymbol{s}_{1}=\left(\delta s_{1}, \delta s_{1}^{\dagger}\right)$ and $\delta \boldsymbol{s}_{2}=\left(\delta s_{2}, \delta s_{2}^{\dagger}\right)$ be two normalized test shifts, i. e., suppose that $\delta s_{\alpha}(y) \cdot \delta s_{\alpha}^{\dagger}(y)=1$ at every $y$ for $\alpha=1,2$. We recall from Lemma 1 in [2, p. 24] that the values of Schouten brackets in (11) are independent of a concrete choice of the normalized functional coefficients $\delta s_{\alpha}$ and $\delta s_{\alpha}^{\dagger}$, which implies that the test shifts $\delta \boldsymbol{s}_{1}$ and $\delta \boldsymbol{s}_{2}$ in the inner and outer brackets can be swapped (this would amount to relabelling $y \rightleftarrows z$ of their arguments, see (21) and (3) on p. (4). In the same way as we did in Example 2.4 in [2], we now do not write the basic (co)vectors $\vec{e}(y)$ and $\vec{e}^{\dagger}(y)$ in expansions of the test shifts and differentials of densities of the functionals (see [2, §2.2-3] for detail); it is enough to know the couplings' values, which are \pm 1 .

We have that $10 \llbracket G, H \rrbracket=$

$$
\begin{aligned}
\iiint \int \mathrm{d} y_{2} \mathrm{~d} y_{3} \mathrm{~d} x_{2} \mathrm{~d} \operatorname{vol}\left(x_{3}\right) \cdot\{ & \left\langle\left(-\frac{\mathrm{d}}{\mathrm{d} y_{2}}\right)(\underbrace{q_{x_{2}}^{\dagger} \exp \left(q_{x_{2}}\right)}_{x_{2}}) \cdot \frac{\mathrm{d}^{2}}{\mathrm{~d} y_{3}^{2}}(\underbrace{\cos q}_{x_{3}})\right\rangle \cdot \underbrace{\left\langle\delta s\left(y_{2}\right), \delta s^{\dagger}\left(y_{3}\right)\right\rangle}_{+1}+ \\
+ & \langle\left(-\frac{\mathrm{d}}{\mathrm{d} y_{2}}\right)(\underbrace{\exp \left(q_{x_{2}}\right)}_{x_{2}}) \cdot \underbrace{q_{x_{3} x_{3}}^{\dagger} \cdot(-\sin q)}_{x_{3}}\rangle \cdot \underbrace{\left\langle\delta s^{\dagger}\left(y_{2}\right), \delta s\left(y_{3}\right)\right\rangle}_{-1}\} ;
\end{aligned}
$$

as usual, we display the integration variables $x_{i}$ under the remnants of respective densities. Next, we obtain that $\llbracket F, \llbracket G, H \rrbracket \rrbracket=$

$$
\begin{aligned}
& \int \mathrm{d} z_{1} \int \mathrm{d} z_{23} \int \mathrm{d} y_{2} \int \mathrm{d} y_{3} \int \mathrm{d} x_{1} \int \mathrm{d} x_{2} \int \mathrm{d} \operatorname{vol}\left(x_{3}\right) \cdot \underbrace{\left\langle\delta s\left(z_{1}\right), \delta s^{\dagger}\left(z_{23}\right)\right\rangle}_{+1} \cdot
\end{aligned}
$$

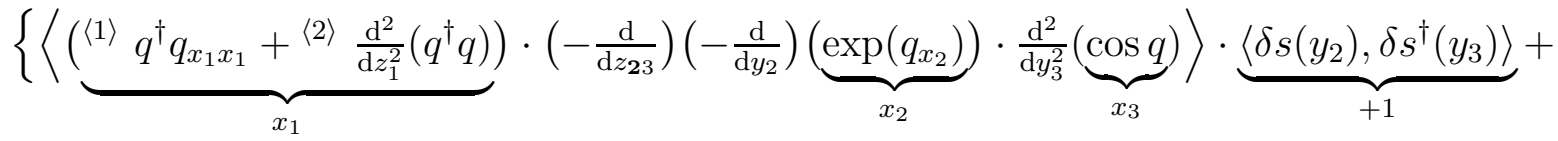

$$
\begin{aligned}
& +\left\langle(\underbrace{(3\rangle q^{\dagger} q_{x_{1} x_{1}}+{ }^{\langle 4\rangle} \frac{\mathrm{d}^{2}}{\mathrm{~d} z_{1}^{2}}\left(q^{\dagger} q\right)}_{x_{1}}) \cdot\left(-\frac{\mathrm{d}}{\mathrm{d} y_{2}}\right)(\underbrace{\exp \left(q_{x_{2}}\right)}_{x_{2}}) \cdot \frac{\mathrm{d}^{2}}{\mathrm{~d} z_{23}^{2}}(\underbrace{-\sin q}_{x_{3}})\right\rangle \cdot\langle\underbrace{\left.\delta \delta s^{\dagger}\left(y_{2}\right), \delta s\left(y_{3}\right)\right\rangle}_{-1}\}+ \\
& +\int \mathrm{d} z_{1} \int \mathrm{d} z_{23} \int \mathrm{d} y_{2} \int \mathrm{d} y_{3} \int \mathrm{d} x_{1} \int \mathrm{d} x_{2} \int \mathrm{d} \operatorname{vol}\left(x_{3}\right) \cdot \underbrace{\left\langle\delta s^{\dagger}\left(z_{1}\right), \delta s\left(z_{23}\right)\right\rangle}_{-1} \cdot \\
& \left\{\left\langle(\underbrace{\langle 5\rangle}_{x_{1}} q q_{x_{1} x_{1}}) \cdot\left(-\frac{\mathrm{d}}{\mathrm{d} z_{23}}\right)\left(-\frac{\mathrm{d}}{\mathrm{d} y_{2}}\right)(\underbrace{q_{x_{2}}^{\dagger} \exp \left(q_{x_{2}}\right)}_{x_{2}}) \cdot \frac{\mathrm{d}^{2}}{\mathrm{~d} y_{3}^{2}}(\underbrace{\cos q}_{x_{3}})+\right.\right. \\
& \left.+(\underbrace{\langle 6\rangle}_{x_{1}} q q_{x_{1} x_{1}}) \cdot\left(-\frac{\mathrm{d}}{\mathrm{d} y_{2}}\right)(\underbrace{q_{x_{2}}^{\dagger} \exp \left(q_{x_{2}}\right)}_{x_{2}}) \cdot \frac{\mathrm{d}^{2}}{\mathrm{~d} y_{3}^{2}}(\underbrace{-\sin q}_{x_{3}})\right\rangle \cdot \underbrace{\left\langle\delta s\left(y_{2}\right), \delta s^{\dagger}\left(y_{3}\right)\right\rangle}_{+1}+
\end{aligned}
$$

\footnotetext{
${ }^{10}$ Let us recall that integrations by parts, which cast the derivatives off the test shifts, are performed only when all the objects - such as the l.-h.s. or r.-h.s. of (1) - are fully composed, all partial derivatives of the functionals' densities are calculated, and reconfigurations of the couplings are ready to start. In practice, this means that partial derivatives like $\vec{\partial} / \partial q_{x}$ or $\overleftarrow{\partial} / \partial q_{x x}^{\dagger}$ dive under $\overrightarrow{\mathrm{d}} / \mathrm{d} y$ or $\overleftarrow{\mathrm{d}} / \mathrm{d} z$ because those total derivatives have not yet appeared at the places where we write them ahead of time.
} 


$$
\begin{aligned}
& +\left\langle(\underbrace{\langle 7\rangle q q_{x_{1} x_{1}}}_{x_{1}}) \cdot\left(-\frac{\mathrm{d}}{\mathrm{d} z_{23}}\right)\left(-\frac{\mathrm{d}}{\mathrm{d} y_{2}}\right)(\underbrace{\exp \left(q_{x_{2}}\right)}_{x_{2}}) \cdot(\underbrace{q_{x_{3} x_{3}}^{\dagger} \cdot(-\sin q)}_{x_{3}})+\right. \\
& \left.+(\underbrace{\langle 8\rangle q q_{x_{1} x_{1}}}_{x_{1}}) \cdot\left(-\frac{\mathrm{d}}{\mathrm{d} y_{2}}\right)(\underbrace{\exp \left(q_{x_{2}}\right)}_{x_{2}}) \cdot(\underbrace{q_{x_{3} x_{3}}^{\dagger} \cdot(-\cos q)}_{x_{3}})\right\rangle \cdot\langle\underbrace{\left.\delta \delta s^{\dagger}\left(y_{2}\right), \delta s\left(y_{3}\right)\right\rangle}_{-1}\} .
\end{aligned}
$$

On the other hand, $\llbracket F, G \rrbracket=$

$$
\begin{aligned}
\iiint \int \mathrm{d} y_{1} \mathrm{~d} y_{2} \mathrm{~d} x_{1} \mathrm{~d} \operatorname{vol}\left(x_{2}\right) \cdot\{ & \left.\langle\underbrace{\left(q^{\dagger} q_{x_{1} x_{1}}+\frac{\mathrm{d}^{2}}{\mathrm{~d} y_{1}^{2}}\left(q^{\dagger} q\right)\right.}_{x_{1}}) \cdot\left(-\frac{\mathrm{d}}{\mathrm{d} y_{2}}\right)(\underbrace{\exp \left(q_{x_{2}}\right)}_{x_{2}})\right\rangle \cdot \underbrace{\left\langle\delta s\left(y_{1}\right), \delta s^{\dagger}\left(y_{2}\right)\right\rangle}_{+1}+ \\
& +\left\langle(\underbrace{q q_{x_{1} x_{1}}}_{x_{1}}) \cdot\left(-\frac{\mathrm{d}}{\mathrm{d} y_{2}}\right)(\underbrace{q_{x_{2}}^{\dagger} \exp \left(q_{x_{2}}\right)}_{x_{2}})\right\rangle \cdot \underbrace{\left\langle\delta s^{\dagger}\left(y_{1}\right), \delta s\left(y_{2}\right)\right\rangle}_{-1}\} .
\end{aligned}
$$

We infer that $\llbracket \llbracket F, G \rrbracket, H \rrbracket=$

$$
\begin{aligned}
& \int \mathrm{d} z_{12} \int \mathrm{d} z_{3} \int \mathrm{d} y_{1} \int \mathrm{d} y_{2} \int \mathrm{d} x_{1} \int \mathrm{d} x_{2} \int \mathrm{d} \operatorname{vol}\left(x_{3}\right) \cdot \underbrace{\left\langle\delta s\left(z_{12}\right), \delta s^{\dagger}\left(z_{3}\right)\right\rangle}_{+1} . \\
& \left\{\left\langle(\underbrace{\langle 9\rangle \frac{\mathrm{d}^{2}}{\mathrm{~d} z_{12}^{2}}\left(q^{\dagger}\right)+{ }^{\langle 10\rangle} \frac{\mathrm{d}^{2}}{\mathrm{~d} y_{1}^{2}}\left(q^{\dagger}\right)}_{x_{1}}) \cdot\left(-\frac{\mathrm{d}}{\mathrm{d} y_{2}}\right)(\underbrace{\exp \left(q_{x_{2}}\right)}_{x_{2}}) \cdot \frac{\mathrm{d}^{2}}{\mathrm{~d} z_{3}^{2}}(\underbrace{\cos q}_{x_{3}})+\right.\right. \\
& \left.+(\underbrace{\langle 1\rangle q^{\dagger} q_{x_{1} x_{1}}+{ }^{\langle 2\rangle} \frac{\mathrm{d}^{2}}{\mathrm{~d} y_{1}^{2}}\left(q^{\dagger} q\right)}_{x_{1}}) \cdot\left(-\frac{\mathrm{d}}{\mathrm{d} z_{12}}\right)\left(-\frac{\mathrm{d}}{\mathrm{d} y_{2}}\right)(\underbrace{\exp \left(q_{x_{2}}\right)}_{x_{2}}) \cdot \frac{\mathrm{d}^{2}}{\mathrm{~d} z_{3}^{2}}(\underbrace{\cos q}_{x_{3}})\right\rangle \cdot \underbrace{\left\langle\delta s\left(y_{1}\right), \delta s^{\dagger}\left(y_{2}\right)\right\rangle}_{+1}+ \\
& +\left\langle(\underbrace{\langle 11\rangle q_{x_{1} x_{1}}+{ }^{\langle 12\rangle} \frac{\mathrm{d}^{2}}{\mathrm{~d} z_{12}^{2}}(q)}_{x_{1}}) \cdot\left(-\frac{\mathrm{d}}{\mathrm{d} y_{2}}\right)(\underbrace{q_{x_{2}}^{\dagger} \exp \left(q_{x_{2}}\right)}_{x_{2}}) \cdot \frac{\mathrm{d}^{2}}{\mathrm{~d} z_{3}^{2}}(\underbrace{\cos q}_{x_{3}})+\right. \\
& \left.+(\underbrace{\langle 5\rangle q q_{x_{1} x_{1}}}_{x_{1}}) \cdot\left(-\frac{\mathrm{d}}{\mathrm{d} z_{12}}\right)\left(-\frac{\mathrm{d}}{\mathrm{d} y_{2}}\right)(\underbrace{q_{x_{2}}^{\dagger} \exp \left(q_{x_{2}}\right)}_{x_{2}}) \cdot \frac{\mathrm{d}^{2}}{\mathrm{~d} z_{3}^{2}}(\underbrace{\cos q}_{x_{3}})\right\rangle \cdot \underbrace{\left\langle\delta s^{\dagger}\left(y_{1}\right), \delta s\left(y_{2}\right)\right\rangle}_{-1}\}+ \\
& +\int \mathrm{d} z_{12} \int \mathrm{d} z_{3} \int \mathrm{d} y_{1} \int \mathrm{d} y_{2} \int \mathrm{d} x_{1} \int \mathrm{d} x_{2} \int \mathrm{d} \operatorname{vol}\left(x_{3}\right) \cdot \underbrace{\left\langle\delta s^{\dagger}\left(z_{12}\right), \delta s\left(z_{3}\right)\right\rangle}_{-1} \cdot
\end{aligned}
$$

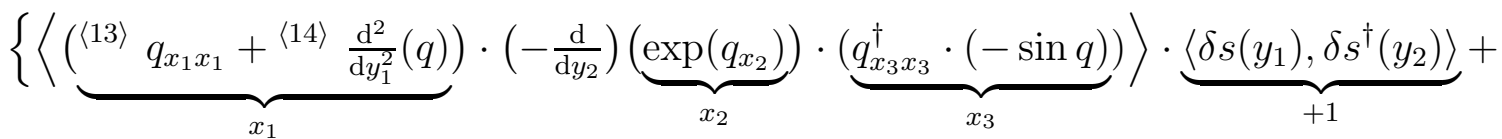

$$
\begin{aligned}
& +\left\langle(\underbrace{\langle 7\rangle q q_{x_{1} x_{1}}}_{x_{1}}) \cdot\left(-\frac{\mathrm{d}}{\mathrm{d} z_{12}}\right)\left(-\frac{\mathrm{d}}{\mathrm{d} y_{2}}\right)(\underbrace{\exp \left(q_{x_{2}}\right)}_{x_{2}}) \cdot(\underbrace{q_{x_{3} x_{3}}^{\dagger} \cdot(-\sin q)}_{x_{3}})\right\rangle \cdot \underbrace{\left\langle\delta s^{\dagger}\left(y_{1}\right), \delta s\left(y_{2}\right)\right\rangle}_{-1}\} .
\end{aligned}
$$

Thirdly, $\llbracket F, H \rrbracket=$

$$
\begin{aligned}
\iiint \int \mathrm{d} y_{1} \mathrm{~d} y_{3} \mathrm{~d} x_{1} \mathrm{~d} \operatorname{vol}\left(x_{3}\right) \cdot\{\langle & \left.\langle\underbrace{\left(q^{\dagger} q_{x_{1} x_{1}}+\frac{\mathrm{d}^{2}}{\mathrm{~d} y_{1}^{2}}\left(q^{\dagger} q\right)\right.}_{x_{1}}) \cdot \frac{\mathrm{d}^{2}}{\mathrm{~d} y_{3}^{2}}(\underbrace{\cos q}_{x_{3}})\right\rangle \cdot \underbrace{\left\langle\delta s\left(y_{1}\right), \delta s^{\dagger}\left(y_{3}\right)\right\rangle}_{+1}+ \\
& +\left\langle(\underbrace{q q_{x_{1} x_{1}}}_{x_{1}}) \cdot(\underbrace{q_{x_{3} x_{3}}^{\dagger} \cdot(-\sin q)}_{x_{3}})\right\rangle \cdot \underbrace{\left\langle\delta s^{\dagger}\left(y_{1}\right), \delta s\left(y_{3}\right)\right\rangle}_{-1}\} .
\end{aligned}
$$


In view of the functionals' gradings, we have $+1 \cdot \llbracket G, \llbracket F, H \rrbracket \rrbracket=$

$$
\begin{aligned}
& \int \mathrm{d} z_{2} \int \mathrm{d} z_{13} \int \mathrm{d} y_{1} \int \mathrm{d} y_{3} \int \mathrm{d} x_{1} \int \mathrm{d} x_{2} \int \mathrm{d} \operatorname{vol}\left(x_{3}\right) \cdot \underbrace{\left\langle\delta s\left(z_{2}\right), \delta s^{\dagger}\left(z_{13}\right)\right\rangle}_{+1} \cdot \\
& \{\left\langle\left(-\frac{\mathrm{d}}{\mathrm{d} z_{2}}\right)(\underbrace{q_{x_{2}}^{\dagger} \exp \left(q_{x_{2}}\right)}_{x_{2}}) \cdot(\underbrace{\langle 11\rangle q_{x_{1} x_{1}}+{ }^{\langle 12\rangle} \frac{\mathrm{d}^{2}}{\mathrm{~d} y_{1}^{2}}(q)}_{x_{1}}) \cdot \frac{\mathrm{d}^{2}}{\mathrm{~d} y_{3}^{2}}(\underbrace{\cos q}_{x_{3}})\right\rangle \cdot \underbrace{\left\langle\delta s\left(y_{1}\right), \delta s^{\dagger}\left(y_{3}\right)\right\rangle}_{+1}+ \\
& +\left\langle\left(-\frac{\mathrm{d}}{\mathrm{d} z_{2}}\right)(\underbrace{q_{x_{2}}^{\dagger} \exp \left(q_{x_{2}}\right)}_{x_{2}}) \cdot(\underbrace{\langle 6\rangle q q_{x_{1} x_{1}}}_{x_{1}}) \cdot \frac{\mathrm{d}^{2}}{\mathrm{~d} z_{13}^{2}}(\underbrace{-\sin q}_{x_{3}})\right\rangle \cdot\langle\underbrace{\left\langle\delta s^{\dagger}\left(y_{1}\right), \delta s\left(y_{3}\right)\right\rangle}_{-1}\}+ \\
& +\int \mathrm{d} z_{2} \int \mathrm{d} z_{13} \int \mathrm{d} y_{1} \int \mathrm{d} y_{3} \int \mathrm{d} x_{1} \int \mathrm{d} x_{2} \int \mathrm{d} \operatorname{vol}\left(x_{3}\right) \cdot \underbrace{\left\langle\delta s^{\dagger}\left(z_{2}\right), \delta s\left(z_{13}\right)\right\rangle}_{-1} \cdot \\
& \left\{\left\langle\left(-\frac{\mathrm{d}}{\mathrm{d} z_{2}}\right)(\underbrace{\exp \left(q_{x_{2}}\right)}_{x_{2}}) \cdot(\underbrace{\langle 10\rangle \frac{\mathrm{d}^{2}}{\mathrm{~d} z_{13}^{2}}\left(q^{\dagger}\right)+{ }^{\langle 9\rangle} \frac{\mathrm{d}^{2}}{\mathrm{~d} y_{1}^{2}}\left(q^{\dagger}\right)}_{x_{1}}) \cdot \frac{\mathrm{d}^{2}}{\mathrm{~d} y_{3}^{2}}(\underbrace{\cos q}_{x_{3}})+\right.\right.
\end{aligned}
$$

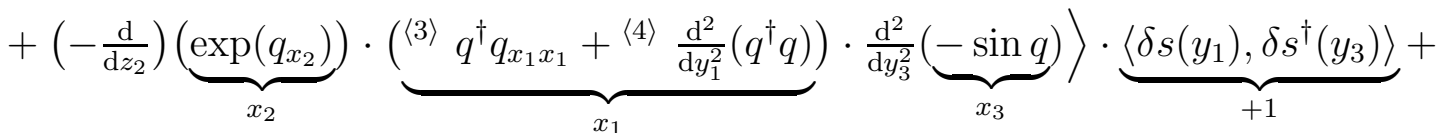

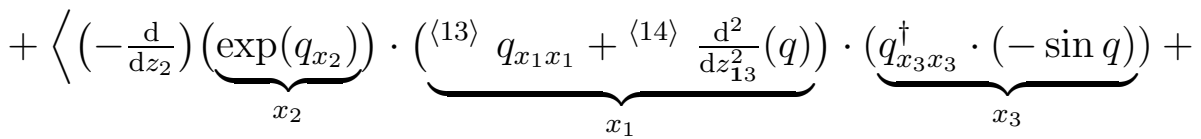

$$
\begin{aligned}
& \left.+\left(-\frac{\mathrm{d}}{\mathrm{d} z_{2}}\right)(\underbrace{\exp \left(q_{x_{2}}\right)}_{x_{2}}) \cdot(\underbrace{\langle 8\rangle q q_{x_{1} x_{1}}}_{x_{1}}) \cdot(\underbrace{q_{x_{3} x_{3}}^{\dagger} \cdot(-\cos q)}_{x_{3}})\right\rangle \cdot \underbrace{\left\langle\delta s^{\dagger}\left(y_{1}\right), \delta s\left(y_{3}\right)\right\rangle}_{-1}\} .
\end{aligned}
$$

Each term $\langle 1\rangle-\langle 8\rangle$ meets its match in the other side of (1), whereas terms $\langle 9\rangle-\langle 14\rangle$ occur in pairs of opposite signs; therefore, they all cancel out in the r.-h.s. of the Jacobi identity. 